\title{
Income and Mental Well-Being of Middle-Aged and Older Americans: Immigrants' Diminished Returns
}

\author{
Shervin Assari ${ }^{*}$ \\ ${ }^{1}$ Department of Family Medicine, Charles R. Drew University of Medicine and Science, Los Angeles, USA \\ Corresponding Author: Shervin Assari, MD, MPH, Assistant Professor, Department of Family Medicine, Charles R. Drew \\ University of Medicine and Science, Los Angeles, USA. Tel.: +1-7342320445, Fax: +1-7346158739, Email: assari@umich.edu
}

Received October 18, 2019; Accepted February 22, 2020; Online Published March 9, 2020

\begin{abstract}
Introduction: Although income is among the major social determinants of mental health of middle-aged and older individuals, socially marginalized groups gain less health from their income and other socioeconomic status (SES) resources compared to socially privileged groups. This pattern is called marginalization-related diminished returns (MDRs). Most of the existing knowledge on MDRs, however, has been derived from studies that have defined marginalization based on race, ethnicity, or sexual orientation. As a result, very limited information exists on whether similar MDRs can be observed for middle-aged and older immigrants or not. Building on the MDRs framework, this study compared a national sample of immigrants and non-immigrants for the effects of income on the mental well-being of middle-aged and older adults in the United States.

Methods: This is a cross-sectional study. The 2015 National Health Interview Survey (NHIS) enrolled 14149 middle-aged and older individuals who were either immigrants $(n=1977 ; 14.0 \%)$ or non-immigrants $(n=12166 ; 86.0 \%)$. The independent variable (IV) was income that was treated as a continuous variable. The dependent variable was mental well-being, also treated as a continuous variable. Age, gender, race, ethnicity, education, marital status, employment, self-rated health, obesity, and region were confounders. Immigration (nativity status) was the moderator. Logistic regression was applied for data analysis.

Results: High income was associated with higher odds of good mental well-being in middle-aged and older adults. However, immigration showed a significant statistical interaction with income, which was suggestive of a smaller protective effect of high income on mental well-being for immigrant than non-immigrant middle-aged and older adults.

Conclusion: In line with MDRs, the association between income and mental well-being is weaker for immigrant than non-immigrant middle-aged and older adults. There is a need to help high income immigrants secure health outcomes similar to those of non-immigrants. Such changes may require bold and innovative economic, public, and social policies that help immigrants more effectively translate their income and socioeconomic resources into tangible outcomes such as mental well-being.

Keywords: Population Groups, Immigrants, Socioeconomic Status, Income, Elderly, Psychological Factors
\end{abstract}

Citation: Assari S. Income and mental well-being of middle-aged and older Americans: immigrants' diminished returns. Int J Travel Med Glob Health. 2020;8(1):37-43. doi:10.34172/ijtmgh.2020.06.

\section{Introduction}

Extensive theoretical and empirical work has established a connection between socioeconomic status (SES) indicators, such as income, and a wide range of physical and mental health outcomes. ${ }^{1-3}$ High income is associated with better mental well-being and happiness ${ }^{4}$ and less depression, ${ }^{5}$ anxiety, ${ }^{6}$ suicidal ideas, ${ }^{7}$ and psychological distress. ${ }^{8,9}$ Similar to other types of social marginalization (e.g., based on race, ethnicity, and sexual orientation), immigration may also reduce the salience of SES indicators such as income on the mental well-being of populations. ${ }^{10-13}$

Sub-populations, however, unequally receive mental health gains from their SES indicators ${ }^{14-20}$ such as income. According to the marginalization-related diminished returns (MDRs) theory ${ }^{21,22}$ the effects of income, ${ }^{23-27}$ educational attainment, ${ }^{28}$ employment, ${ }^{29,30}$ and marital status on mental, ${ }^{31,32}$ behavioral, ${ }^{28,33-35}$ and physical ${ }^{36-39}$ health outcomes are weaker for racial, ethnic, and sexual minorities than for the majority group. This pattern has been established for Blacks, ${ }^{15,16}$ Hispanics, ${ }^{23,40}$ Asian Americans, ${ }^{41}$ Native Americans, ${ }^{42}$ as well as lesbian, gay, bisexual, and transgender (LGBT) individuals. ${ }^{43-45}$ For example, high income Black men are at an increased rather than reduced risk of depression. Similarly, high SES LGBTs remain at high risk of poor mental health, ${ }^{43}$ smoking, ${ }^{44}$ and obesity. ${ }^{45}$

While according to the MDRs literature, racial, ethnic, and sexual minority status are all associated with diminished health returns of income, ${ }^{23-27}$ educational attainment, ${ }^{36}$ occupation, ${ }^{29,30}$ and marital status, ${ }^{46}$ it is yet unknown whether the same MDRs may also be relevant to immigrants. In other

Copyright $\odot 2020$ The Author(s). This is an open-access article distributed under the terms of the Creative Commons Attribution License (http:// creativecommons.org/licenses/by/4.0), which permits unrestricted use, distribution, and reproduction in any medium, provided the original work is properly cited. 
words, it remains unclear if immigrants gain less health from their SES resources, such as income, compared to nonimmigrant people. Similar to other minority groups based on race, ${ }^{31}$ ethnicity, ${ }^{23,34}$ and sexual minority status, ${ }^{43-45}$ immigrants are also marginalized by the host society. Immigrants are being widely discriminated against and are treated differently than the native people by various social institutions. ${ }^{47-51}$ Immigrants also experience a considerable amount of stigma and may internalize hopelessness and sadness.

Borrowing data from the National Health Interview Survey (NHIS), this study was conducted to compare immigrants and non-immigrants for the association between income and mental well-being of middle-aged and older adults in the US. Built on MDRs, immigration status was conceptualized as a marginalizing identity. Thus, similar to the pattern observed for Blacks, Hispanics, Asian Americans, Native Americans, and LGBTs, it was expected weaker effects of income on the psychological well-being of immigrant rather than nonimmigrant middle-aged and older people.

\section{Methods}

This cross-sectional study used data from the 2015 NHIS. The NHIS is the primary source of information regarding the physical health status of American adults 18 years or older. The NHIS sample is composed of U.S. residents, civilians, and noninstitutionalized people. The current analysis is, however, limited to middle-aged and older adults.

\section{Participants and Sampling}

The NHIS used a multi-stage clustered/stratified random sampling. First, 428 primary sampling units (PSUs) drawn from 1900 geographically defined PSUs were sampled. All 50 U.S. states and the District of Columbia that have representatives had PSUs in the sample. The PSUs were either a metropolitan statistical area, a single county, or a small group of contiguous counties. For this analysis, only data regarding middle-aged and older adults was used.

\section{Process}

Data is collected by the National Center for Health Statistics (NCHS), which is a part of the Centers for Disease Control and Prevention (CDC), and by the U.S. Census Bureau via face-to-face interviews in participants' households. On some occasions, this face-to-face interview is followed or replaced by a telephone interview.

\section{Participants}

The total sample in this study was 14149 middle-aged and older adults who were either immigrants $(\mathrm{n}=1977 ; 14.0 \%)$ or non-immigrants $(n=12166 ; 86.0 \%)$. People could be of any race/ethnicity to be included in this study. There was no exclusion criterion.

\section{Measures}

\section{Predictor}

Income. Annual income was self-reported. This variable was measured in dollars as a continuous variable. For the current analysis, however, a dichotomous variable with a cut point of 35000 USD was used. Individuals with high income were coded as 1 , and those with lower income were coded as 0 .

\section{Moderator}

Immigration status. Nativity was self-reported. All participants were asked if they were born in the U.S. The responses were coded 1 for immigrants and 0 for non-immigrants.

\section{Covariates}

Sociodemographic factors. Demographic factors included age, gender, region, race, ethnicity, education, marital status, and employment. Age (years) was a continuous variable. Gender was a dichotomous measure (male $=1$, female $=0$ ). The region was either Northeast, Midwest, South, or West. Participants self-identified their race and ethnicity, which were both operationalized as categorical variables. Race was White only (reference category), Black/African American only, Native American/Alaska Native only, Asian only, Multiple race, and race group not releasable (masked or missing). Ethnicity was Hispanics $=1$, Non-Hispanics $=0$ (reference category). Educational attainment was operationalized as a continuous variable ranging from 0 to 24 . Participants were asked about the number of years of schooling. Marital status was a dichotomous variable with married as 1 . Employment status was a dichotomous variable with employed as 1 .

Health confounders included self-rated health (SRH) and obesity.

Self-rated Health (SRH). The conventional single item of SRH was used to measure overall health. Responses were excellent, very good, good, fair, and poor. Responses were dichotomized so fair and poor reflected poor and other responses reflected good health. Poor SRH is shown to predict outcomes including mortality in clinical as well as community samples. ${ }^{52}$

Obesity. Obesity was defined based on the body mass index, which was calculated based on self-reported height and weight. A BMI equal or larger than 30 was considered as obesity. This variable was treated as a dichotomous variable.

\section{Dependent Variable}

Psychological Well-being. The following items were used to measure psychological well-being. 1) 1) How often you felt so sad nothing cheers you up during the past 30 days, 2) How often you felt nervous during the past 30 days, 3) How often you felt restless/fidgety, past 30 days, 4) How often you felt hopeless during the past 30 days, 5) How often you felt everything was an effort during the past 30 days, and 6) How often you felt worthless during the past 30 days. Responses to these items included 0) all of the time, 1) most of the time, 2) some of the time, 3) a little of the time, and 4) none of the time. A sum score was calculated, ranging from 0 to 24 , with a higher score indicating higher psychological well-being (lack of distress). This measure was operationalized as a categorical variable. A cut of 22 was considered to define good versus poor mental well-being. These items are based mainly on the Kessler 6-item Psychological Distress measure, which is 
widely validated and used. ${ }^{53}$

Statistical Analyses

Given the NHIS's multi-stage sampling design, it was necessary to apply SPSS 23.0 (IBM Inc., NY, USA) for data analysis. Using SPSS, weights that were due to the design variables (strata, clusters, and non-response) were adjusted for the NHIS survey. Taylor series linearization was applied for the re-estimation of the standard errors (SEs). For descriptive statistics, weighted means and frequencies were used.

For our multivariable analyses, four logistic regression models were applied. In these models, income was the independent variable, psychological well-being was the dependent variable, demographic factors and education, ethnicity, and region were the control variables, and immigration status was the moderator. The first two models were calculated in the pooled sample that included both immigrants and non-immigrants. Model 1 did not include immigration by income interaction terms. Model 2, however, included immigration by income interaction terms. Model 3 and Model 4 were performed in non-immigrants and immigrants, respectively. Adjusted odds ratio (OR), 95\% confidence intervals (CI), SE, and $P$ values were reported. A $P$ value of less than 0.05 was considered significant.

\section{Results}

Descriptive Statistics

The total sample in this study was 14149 immigrant and non-immigrant middle-aged and older (55+ years) American adults. Table 1 depicts the descriptive statistics of the participants overall and based on nativity.

\section{Pooled Sample Logistic Regressions}

Table 2 shows the results of two logistic regressions in the pooled sample with income as the predictor and psychological well-being as the outcome (dependent variable). Model 1 only included the main effects; however, Model 2 added the interaction terms between immigration status and income. Based on Model 1, high income was positively associated with psychological well-being. Model 2, however, revealed a statistically significant interaction between income and immigration on the psychological well-being of middle-aged and older adults. The model suggested that the protective effect of income against psychological well-being is smaller for immigrant than non-immigrant middle-aged and older adults (Table 2).

\section{Stratified Logistic Regressions}

Table 3 shows the results of two logistic regressions on nonimmigrants (Model 3) and one on immigrants (Model 4) for each outcome. In these models, income was the predictor and psychological well-being was the outcome (dependent variable). Based on Model 3, high income was positively associated with psychological well-being for non-immigrant middle-aged and older adults. Model 3 did not show income as a predictor of psychological well-being for immigrant middle-aged and older adults (Table 3 ).
Table 1. Descriptive Statistics Overall and Based on Immigration ( $\mathrm{n}=14149)$

\begin{tabular}{|c|c|c|c|c|c|c|}
\hline & \multicolumn{2}{|l|}{ All } & \multicolumn{2}{|c|}{ Non-Immigrant } & \multicolumn{2}{|c|}{ Immigrant } \\
\hline & No. & $\%$ & No. & $\%$ & No. & $\%$ \\
\hline \multicolumn{7}{|l|}{ Immigrants } \\
\hline No & 12166 & 86.0 & 12166 & 100.0 & - & - \\
\hline Yes & 1977 & 14.0 & - & - & 1977 & 100.0 \\
\hline \multicolumn{7}{|l|}{ Ethnicity* } \\
\hline Non-Hispanic & 12698 & 89.7 & 11668 & 95.9 & 1026 & 51.9 \\
\hline Hispanic & 1451 & 10.3 & 498 & 4.1 & 951 & 48.1 \\
\hline \multicolumn{7}{|l|}{ Race* } \\
\hline White only & 11351 & 80.2 & 10047 & 82.6 & 1298 & 65.7 \\
\hline Black/African American only & 1843 & 13.0 & 1671 & 13.7 & 172 & 8.7 \\
\hline AIAN only & 123 & 0.9 & 98 & 0.8 & 25 & 1.3 \\
\hline Asian only & 599 & 4.2 & 148 & 1.2 & 451 & 22.8 \\
\hline Multiple race & 218 & 1.5 & 194 & 1.6 & 24 & 1.2 \\
\hline Race group not releasable & 15 & 0.1 & 8 & 0.1 & 7 & 0.4 \\
\hline \multicolumn{7}{|l|}{ Gender } \\
\hline Female & 8079 & 57.1 & 6917 & 56.9 & 1159 & 58.6 \\
\hline Male & 6070 & 42.9 & 5249 & 43.1 & 818 & 41.4 \\
\hline \multicolumn{7}{|l|}{ Education $(y)^{*}$} \\
\hline Less than 12 & 2257 & 16.1 & 1590 & 13.1 & 666 & 34.2 \\
\hline 12 & 3898 & 27.7 & 3476 & 28.7 & 421 & 21.6 \\
\hline $13-15$ & 3519 & 25.0 & 3210 & 26.5 & 309 & 15.9 \\
\hline $16+$ & 4388 & 31.2 & 3834 & 31.7 & 550 & 28.3 \\
\hline \multicolumn{7}{|l|}{ Region* } \\
\hline Northeast & 2531 & 17.9 & 2030 & 16.7 & 501 & 25.3 \\
\hline Midwest & 3002 & 21.2 & 2836 & 23.3 & 166 & 8.4 \\
\hline South & 4881 & 34.5 & 4262 & 35.0 & 617 & 31.2 \\
\hline West & 3735 & 26.4 & 3038 & 25.0 & 693 & 35.1 \\
\hline \multicolumn{7}{|l|}{ Marital status* } \\
\hline Non-Married & 7769 & 54.9 & 6777 & 55.7 & 990 & 50.1 \\
\hline Married & 6380 & 45.1 & 5389 & 44.3 & 987 & 49.9 \\
\hline \multicolumn{7}{|l|}{ Employed } \\
\hline No & 9759 & 69.0 & 8429 & 69.3 & 1326 & 67.1 \\
\hline Yes & 4390 & 31.0 & 3737 & 30.7 & 651 & 32.9 \\
\hline \multicolumn{7}{|l|}{$\mathrm{BMI} \geq 30^{*}$} \\
\hline No & 9372 & 66.2 & 7951 & 65.4 & 1418 & 71.7 \\
\hline Yes & 4777 & 33.8 & 4215 & 34.6 & 559 & 28.3 \\
\hline \multicolumn{7}{|l|}{ SRH; Poor* } \\
\hline No & 11065 & 78.2 & 9655 & 79.4 & 1406 & 71.2 \\
\hline Yes & 3076 & 21.8 & 2504 & 20.6 & 570 & 28.8 \\
\hline \multicolumn{7}{|l|}{ Income $>35 \mathrm{k}^{*}$} \\
\hline No & 2137 & 47.9 & 1790 & 46.8 & 347 & 54.8 \\
\hline Yes & 2324 & 52.1 & 2038 & 53.2 & 286 & 45.2 \\
\hline \multicolumn{7}{|l|}{ Mental Well-being } \\
\hline No & 4073 & 30.0 & 3485 & 29.8 & 588 & 31.5 \\
\hline \multirow[t]{2}{*}{ Yes } & 9482 & 70.0 & 8195 & 70.2 & 1281 & 68.5 \\
\hline & Mean & SD & Mean & SD & Mean & SD \\
\hline Age* & 68.17 & 9.09 & 68.28 & 9.13 & 67.55 & 8.80 \\
\hline
\end{tabular}


Table 2. Logistic Regressions in the Pooled Sample ( $\mathrm{n}=14149)$

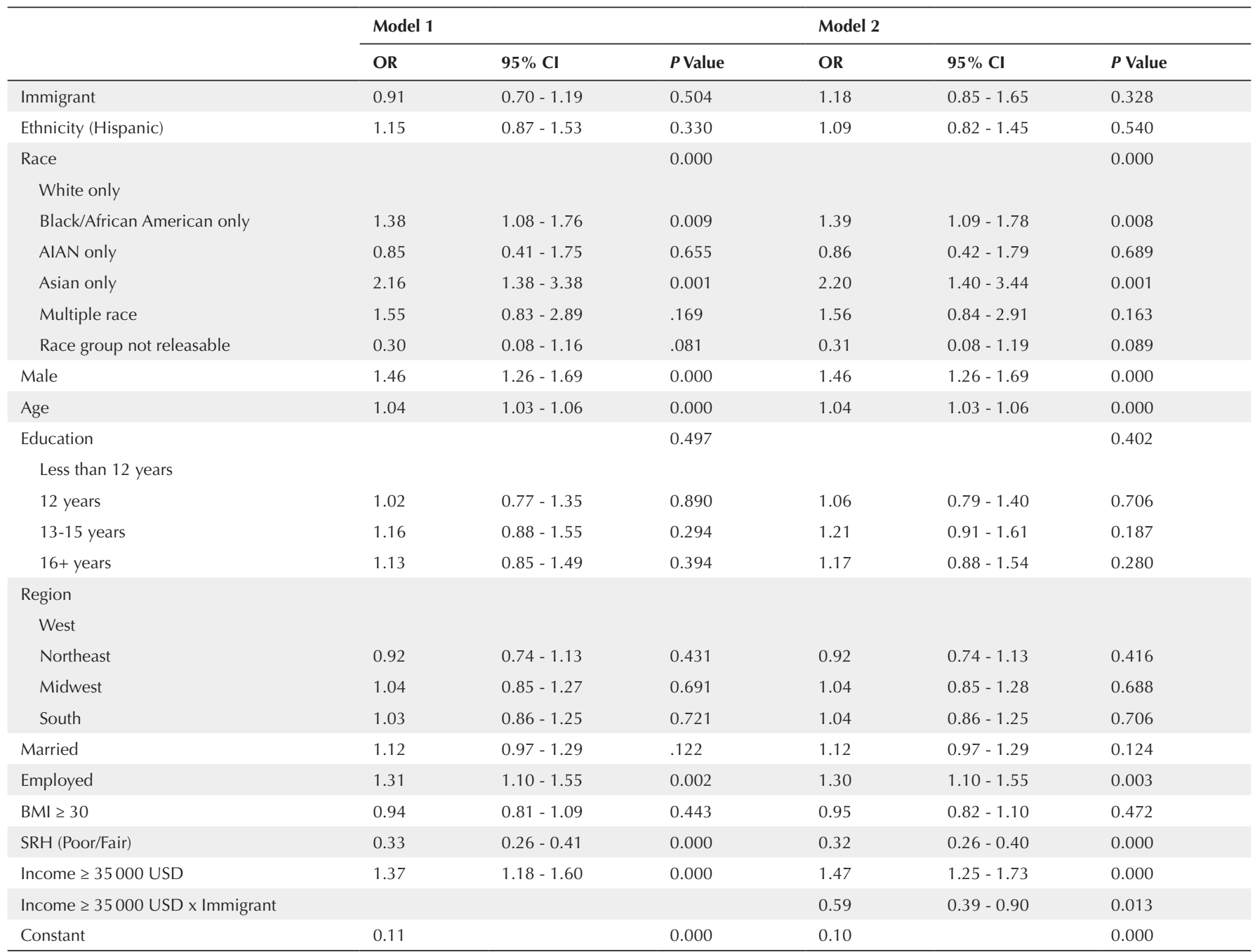

Abbreviation: SRH, self-rated health; BMI, body mass index.

\section{Discussion}

The current study supports the finding that income is associated with better psychological well-being in middleaged and older adults; however, this effect is larger for nonimmigrants than for immigrants.

Marginalization, broadly defined, reduces the health return of income and SES indicators. This is supported by the observations that Blacks, ${ }^{31}$ Hispanics ${ }^{23,34}$ Asian Americans, ${ }^{41}$ Native Americans, ${ }^{42}$ and LGBTs ${ }^{45}$ show MDRs. If any type of marginalization reduces the health gains that follow SES indicators, such as income, similar patterns for immigrants should be expected. ${ }^{47-51}$ Societal and structural factors such as social stratification, residential segregation, labor market discrimination, and low availability of resources in urban areas may all suggest that immigrants may be less likely to fully benefit from their income.

The observation that as income increases, the psychological well-being of middle-aged and older adults improves, but less for immigrants than non-immigrants, is an extension of previous literature on $\mathrm{MDRs}^{21,22}$ of income, ${ }^{23-27}$ educational attainment, ${ }^{36}$ occupation, ${ }^{29,30}$ and marital status ${ }^{46}$ on a wide range of mental health outcomes, including psychological distress, ${ }^{54}$ depression, ${ }^{24,55}$ suicide, ${ }^{31}$ and anxiety. ${ }^{46}$ These MDRs go beyond physical health outcomes and extend to behavioral outcomes such as vaping, ${ }^{33}$ smoking, ${ }^{35,42,44,56,57}$ drinking, ${ }^{34,58}$ diet, ${ }^{59}$ and exercise. ${ }^{11}$ Finally, MDRs are seen for physical health outcomes such as obesity, ${ }^{36,37}$ self-rated health, ${ }^{23,32,54}$ CMCs, ${ }^{25,26,38}$ disability, ${ }^{60}$ and mortality. ${ }^{39}$ Moreover, it is not just income, ${ }^{25}$ educational attainment, ${ }^{32}$ occupation, ${ }^{39}$ and marital status ${ }^{46}$ all generate less health for the majority than for marginalized people. Social marginalization, regardless of its type, whether based on race, ${ }^{61}$ ethnicity, ${ }^{23,34}$ sexual orientation, ${ }^{43-45}$ or immigration, are consistently associated with less health gain from income and other SES indicators. ${ }^{61}$

The robust and consistent nature of MDRs shift the blame from marginalized people to the society that reduces the gain of all vulnerable and disadvantaged groups. Thus, these patterns suggest that they are caused by the function and structure of society. U.S. social institutions differentially treat people based on their color, race, ethnicity, class, heritage, and nativity, which results in the systemic marginalization of non-majority groups. Such marginalization reduces people's chances of full participation and full realization of benefits from the resources that are available to them. The racism, 
Table 3. Logistic Regressions in Non-immigrants and Immigrants ( $n=14149)$

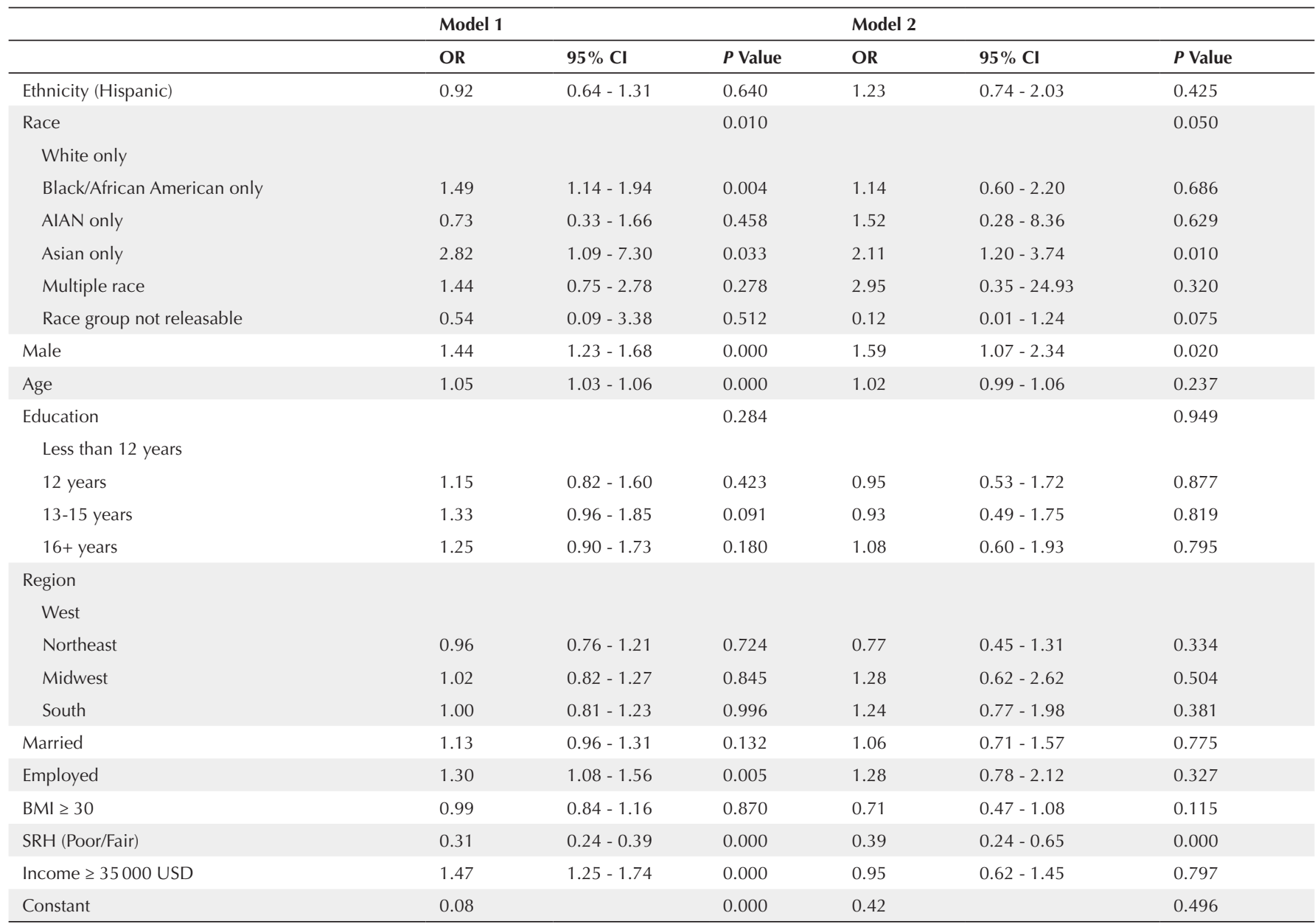

Abbreviation: SRH, self-rated health; BMI, body mass index.

xenophobia, and nationalism embedded in the social fabric of U.S. society reduce the ability of immigrants, LGBTs, and racial and ethnic minorities to fully leverage their human capital and turn them into tangible outcomes. As a result, they show less than expected benefits in the presence of income and other SES resources. ${ }^{21,22}$

\section{Limitations}

The current results should be interpreted with the methodological limitations in mind. First, any cross-sectional study is limited in drawing causal inferences. It cannot be ruled out that excessive health problems would influence social mobility and the ability to generate income. Thus, reverse causality cannot be ruled out in this study. The results should not be interpreted as causation, but as association. Moreover, the mechanisms by which MDRs of income emerge were not investigated in this study. Lower purchasing power of income for immigrants may be the mechanism. Also, the researchers did not have access to country of origin or sources of income; nor did we control for type of occupation, wealth, assets, parental education, or first-generation college student. Future research should replicate and validate these findings using longitudinal data with a more comprehensive list of measures of income, nativity, country of origin and other
SES indicators. Future research may also include contextual factors such as ethnic composition of neighborhoods, SES, or density of resources as factors that may cause MDRs. It is likely that high income immigrants report poor mental health, because they need to spend more time on the job or because they face extra stress in gaining such income. Finally, there is a need to compare immigrants from Asian, African, and Latino countries, as each culture may adopt U.S. culture differently.

\section{Conclusion}

While income enhances the psychological well-being of middle-aged and older adults, this is less true for immigrants than non-immigrants. Thus, health disparities in immigrants are in part due to inequalities in marginal returns of SES indicators such as income. To eliminate health inequality between immigrants and non-immigrants, it is essential to recognize and address MDRs-related inequalities. To undo MDRs, there is a need for bold policies that can equalize the health return of income and other SES indicators across groups. Such policies should go beyond equal access to income to equality in the returns of income and other SES indicators across social groups. Specific policies and programs should help immigrants to more effectively mobilize and leverage 


\section{Research Highlights}

\section{What Is Already Known?}

High income is associated with the better mental wellbeing of individuals. Income, however, may be associated with different levels of mental health gain for population sub-groups.

\section{What This Study Adds?}

In line with the marginalization-related diminished returns, income seems to generate more mental health for non-immigrants that for immigrants in the United States.

their income to gain tangible outcomes. Ways by which the purchasing power of immigrants can be enhanced should be studied in future research.

\section{Conflicts of Interest Disclosures}

The author declares no conflicts of interest.

\section{Ethical Approval}

All participants provided written consent. The NHIS protocol was approved by the CDC Instructional Review Board (IRB). According to the NIH guidelines as well as the decision tool regarding human subject research, secondary analyses of publicly available, fully de-identified existing data is "NonHuman Subject Research." The definition of "Non-Human Subject Research" as well as the decision tool are available here: https://grants.nih.gov/policy/humansubjects/hsdecision.htm. Non-human subject research is exempt from the IRB review.

\section{Funding/Support}

Assari is supported by the NIH under Awards 54MD008149, R25 MD007610, 2U54MD007598, and U54 TR001627. Assari is also supported by D084526-03 and CA201415-02.

\section{References}

1. Marmot M. Social determinants of health inequalities. Lancet. 2005;365(9464):1099-1104. doi:10.1016/s0140-6736(05)711466.

2. Marmot M. The Status Syndrome: How Social Standing Affects Our Health and Longevity. London: Bloomsbury Press; 2004.

3. Marmot M. Economic and social determinants of disease. Bull World Health Organ. 2001;79(10):988-989.

4. Assari S. Race, education attainment, and happiness in the United States. Int J Epidemiol Res. 2019;6(2):76-82. doi:10.15171/ ijer.2019.14.

5. Hudson DL, Puterman E, Bibbins-Domingo K, Matthews KA, Adler NE. Race, life course socioeconomic position, racial discrimination, depressive symptoms and self-rated health. Soc Sci Med. 2013;97:7-14. doi:10.1016/j.socscimed.2013.07.031.

6. Murcia M, Chastang JF, Niedhammer I. Educational inequalities in major depressive and generalized anxiety disorders: results from the French national SIP study. Soc Psychiatry Psychiatr Epidemiol. 2015;50(6):919-928. doi:10.1007/s00127-015-1010-9.

7. Phillips JA, Hempstead K. Differences in US suicide rates by educational attainment, 2000-2014. Am J Prev Med. 2017;53(4):e123-e130. doi:10.1016/j.amepre.2017.04.010.

8. Merz EC, Tottenham N, Noble KG. Socioeconomic status, amygdala volume, and internalizing symptoms in children and adolescents. J Clin Child Adolesc Psychol. 2018;47(2):312-323. doi:10.1080/15374416.2017.1326122.

9. Ursache A, Merz EC, Melvin S, Meyer J, Noble KG. Socioeconomic status, hair cortisol and internalizing symptoms in parents and children. Psychoneuroendocrinology. 2017;78:142-150. doi:10.1016/j.psyneuen.2017.01.020.

10. Assari S. Socioeconomic determinants of systolic blood pressure; minorities' diminished returns. J Health Econ Dev. 2019;1(1):1-11.

11. Assari S. Educational attainment and exercise frequency in American women; blacks' diminished returns. Womens Health Bull. 2019;6(3). doi:10.5812/whb.87413.

12. Assari S. Ethnicity, educational attainment, and physical health of older adults in the United States. Aging Med (Milton). 2019;2(2):104-111. doi:10.1002/agm2.12050.

13. Assari S. Parental educational attainment and academic performance of American college students; blacks' diminished returns. J Health Econ Dev. 2019;1(1):21-31.

14. Assari S. Cross-country differences in the additive effects of socioeconomics, health behaviors and medical comorbidities on disability among older adults with heart disease. J Tehran Heart Cent. 2015;10(1):24-33.

15. Assari S, Moghani Lankarani M. Does multi-morbidity mediate the effect of socioeconomics on self-rated health? cross-country differences. Int J Prev Med. 2015;6:85. doi:10.4103/20087802.164413 .

16. Assari S. Cross-country variation in additive effects of socioeconomics, health behaviors, and comorbidities on subjective health of patients with diabetes. J Diabetes Metab Disord. 2014;13(1):36. doi:10.1186/2251-6581-13-36.

17. Assari S, Moghani Lankarani R, Moghani Lankarani M. Crosscountry differences in the association between diabetes and disability. J Diabetes Metab Disord. 2014;13(1):3. doi:10.1186/2251-6581-13-3.

18. Hudson DL, Bullard KM, Neighbors HW, Geronimus AT, Yang J, Jackson JS. Are benefits conferred with greater socioeconomic position undermined by racial discrimination among African American men? J Mens Health. 2012;9(2):127-136. doi:10.1016/j. jomh.2012.03.006.

19. Hudson DL, Neighbors HW, Geronimus AT, Jackson JS. The relationship between socioeconomic position and depression among a US nationally representative sample of African Americans. Soc Psychiatry Psychiatr Epidemiol. 2012;47(3):373381. doi:10.1007/s00127-011-0348-x.

20. Hudson DL, Neighbors HW, Geronimus AT, Jackson JS. Racial discrimination, John Henryism, and depression among African Americans. J Black Psychol. 2016;42(3):221-243. doi:10.1177/0095798414567757.

21. Assari S. Health disparities due to diminished return among black Americans: public policy solutions. Soc Issues Policy Rev. 2018;12(1):112-145. doi:10.1111/sipr.12042.

22. Assari S. Unequal gain of equal resources across racial groups. Int J Health Policy Manag. 2018;7(1):1-9. doi:10.15171/ ijhpm.2017.90.

23. Assari S. Socioeconomic status and self-rated oral health; diminished return among Hispanic whites. Dent J (Basel). 2018;6(2). doi:10.3390/dj6020011.

24. Assari S. High income protects whites but not African Americans against risk of depression. Healthcare (Basel). 2018;6(2). doi:10.3390/healthcare6020037.

25. Assari S. The benefits of higher income in protecting against chronic medical conditions are smaller for African Americans than whites. Healthcare(Basel). 2018;6(1). doi:10.3390/healthcare6010002.

26. Assari S, Caldwell $\mathrm{CH}$. Family income at birth and risk of attention deficit hyperactivity disorder at age 15: racial differences. Children (Basel). 2019;6(1). doi:10.3390/children6010010.

27. Assari S, Hani N. Household income and children's unmet dental care need; blacks' diminished return. Dent J (Basel). 2018;6(2). doi:10.3390/dj6020017. 
28. Assari S, Mistry R. Educational attainment and smoking status in a national sample of American adults; evidence for the blacks' diminished return. Int J Environ Res Public Health. 2018;15(4). doi:10.3390/ijerph15040763.

29. Assari S. Life expectancy gain due to employment status depends on race, gender, education, and their intersections. J Racial Ethn Health Disparities. 2018;5(2):375-386. doi:10.1007/s40615-017$0381-x$

30. Assari S, Bazargan M. Unequal associations between educational attainment and occupational stress across racial and ethnic groups. Int J Environ Res Public Health. 2019;16(19). doi:10.3390/ ijerph16193539.

31. Assari S, Schatten HT, Arias SA, Miller IW, Camargo CA, Boudreaux ED. Higher educational attainment is associated with lower risk of a future suicide attempt among non-Hispanic whites but not nonHispanic blacks. J Racial Ethn Health Disparities. 2019;6(5):10011010. doi:10.1007/s40615-019-00601-z.

32. Assari S. Blacks' diminished return of education attainment on subjective health; mediating effect of income. Brain Sci. 2018;8(9). doi:10.3390/brainsci8090176.

33. Assari S, Mistry R, Bazargan M. Race, Educational Attainment, And E-Cigarette Use. J Med Res Innov. 2020;4(1):e000185.

34. Assari S, Farokhnia M, Mistry R. Education attainment and alcohol binge drinking: diminished returns of Hispanics in Los Angeles. Behav Sci (Basel). 2019;9(1). doi:10.3390/bs9010009.

35. Assari S, Mistry R. Diminished return of employment on ever smoking among Hispanic whites in Los Angeles. Health Equity. 2019;3(1):138-144. doi:10.1089/heq.2018.0070.

36. Assari S. Family income reduces risk of obesity for white but not black children. Children (Basel). 2018;5(6). doi:10.3390/ children5060073.

37. Assari S, Thomas A, Caldwell CH, Mincy RB. Blacks' diminished health return of family structure and socioeconomic status; 15 years of follow-up of a national urban sample of youth. J Urban Health. 2018;95(1):21-35. doi:10.1007/s11524-017-0217-3.

38. Assari S, Moghani Lankarani M. Poverty status and childhood asthma in white and black families: National Survey of Children's Health. Healthcare (Basel). 2018;6(2). doi:10.3390/ healthcare6020062.

39. Assari S, Moghani Lankarani M. Race and urbanity alter the protective effect of education but not income on mortality. Front Public Health. 2016;4:100. doi:10.3389/fpubh.2016.00100.

40. Assari S, Bazargan M. Educational attainment and self-rated oral health among American older adults: Hispanics' diminished returns. Dent J (Basel). 2019;7(4). doi:10.3390/dj7040097.

41. Assari S, Boyce S, Bazargan M, Caldwell CH. Mathematical performance of American youth: diminished returns of educational attainment of Asian-American parents. Educ Sci. 2020;10(2):32. doi:10.3390/educsci10020032.

42. Assari S, Bazargan M. Protective Effects of educational attainment against cigarette smoking; diminished returns of American Indians and Alaska natives in the National Health Interview Survey. Int J Travel Med Glob Health. 2019;7(3):105-110. doi:10.15171/ ijtmgh.2019.22.

43. Assari S, Bazargan M. Educational attainment and subjective health and well-being; diminished returns of lesbian, gay, and bisexual individuals. Behav Sci (Basel). 2019;9(9). doi:10.3390/ bs9090090.

44. Assari S, Bazargan M. Education level and cigarette smoking: diminished returns of lesbian, gay and bisexual individuals. Behav
Sci (Basel). 2019;9(10). doi:10.3390/bs9100103.

45. Assari S. Education attainment and obesity: differential returns based on sexual orientation. Behav Sci (Basel). 2019;9(2). doi:10.3390/bs9020016.

46. Assari S, Caldwell $\mathrm{CH}$, Zimmerman MA. Family structure and subsequent anxiety symptoms; minorities' diminished return. Brain Sci. 2018;8(6). doi:10.3390/brainsci8060097.

47. Cobb CL, Meca A, Branscombe NR, et al. Perceived discrimination and well-being among unauthorized Hispanic immigrants: The moderating role of ethnic/racial group identity centrality. Cultur Divers Ethnic Minor Psychol. 2019;25(2):280-287. doi:10.1037/ cdp0000227.

48. Fischer S, Nater UM, Strahler J, et al. Psychobiological impact of ethnic discrimination in Turkish immigrants living in Germany. Stress. 2017;20(2):167-174. doi:10.1080/10253890.2017.12964 30.

49. Flippen CA, Parrado EA. Perceived discrimination among Latino immigrants in new destinations: the case of Durham, NC. Sociol Perspect. 2015;58(4):666-685. doi:10.1177/0731121415574397.

50. Straiton ML, Aambø AK, Johansen R. Perceived discrimination, health and mental health among immigrants in Norway: the role of moderating factors. BMC Public Health. 2019;19(1):325. doi:10.1186/s12889-019-6649-9.

51. Yoo HC, Gee GC, Takeuchi D. Discrimination and health among Asian American immigrants: disentangling racial from language discrimination. Soc Sci Med. 2009;68(4):726-732. doi:10.1016/j. socscimed.2008.11.013.

52. Idler EL, Benyamini Y. Self-rated health and mortality: a review of twenty-seven community studies. J Health Soc Behav. 1997;38(1):21-37. doi:10.2307/2955359.

53. Andrews G, SladeT. Interpreting scores on the Kessler Psychological Distress Scale (K10). Aust N Z J Public Health. 2001;25(6):494497. doi:10.1111/j.1467-842x.2001.tb00310.x.

54. Assari S, Lapeyrouse LM, Neighbors HW. Income and selfrated mental health: diminished returns for high income black Americans. Behav Sci (Basel). 2018;8(5). doi:10.3390/bs8050050.

55. Assari S. Educational attainment better protects African American women than African American men against depressive symptoms and psychological distress. Brain Sci. 2018;8(10). doi:10.3390/ brainsci8100182.

56. Assari S, Bazargan M. Unequal effects of educational attainment on workplace exposure to second-hand smoke by race and ethnicity; minorities' diminished returns in the National Health Interview Survey (NHIS). J Med Res Innov. 2019;3(2). doi:10.32892/jmri.179.

57. Assari S, Bazargan M. Second-hand smoke exposure at home in the United States; minorities' diminished returns. Int J Travel Med Glob Health. 2019;7(4):135-141. doi:10.15171/ijtmgh.2019.28.

58. Assari S, Moghani Lankarani M. Education and alcohol consumption among older Americans; black-white differences. Front Public Health. 2016;4:67. doi:10.3389/fpubh.2016.00067.

59. Assari S, Moghani Lankarani M. Educational attainment promotes fruit and vegetable intake for whites but not blacks. J (Basel). 2018;1(1):29-41. doi:10.3390/j1010005.

60. Assari S, Bazargan M. Educational attainment better reduces disability for non-Hispanic than Hispanic Americans. Eur J Investig Health Psychol Educ. 2020;10(1):10-17. doi:10.3390/ ejihpe10010002.

61. Assari S. Parental educational attainment and mental well-being of college students: diminished returns of Blacks. Brain Sci. 2018;8(11). doi:10.3390/brainsci8110193. 